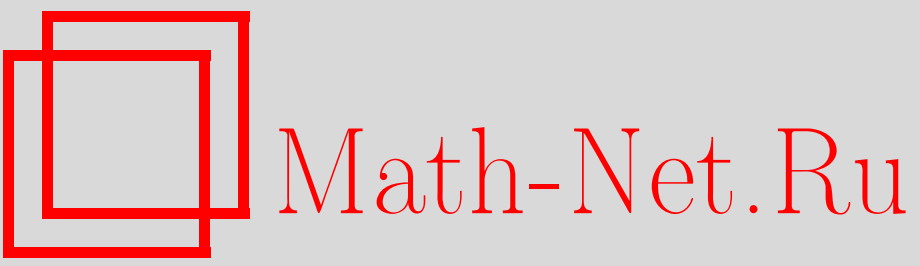

В. А. Вшивцев, А. В. Прокопов, А. В. Татаринцев, Некоторые особенности термодинамики нелинейных классических систем, ТМФ, 2000, том 125, номер 2, 315-325

DOI: https://doi.org/10.4213/tmf671

Использование Общероссийского математического портала Math-Net.Ru подразумевает, что вы прочитали и согласны с пользовательским соглашением

http: //www . mathnet.ru/rus/agreement

Параметры загрузки:

IP : 35.173 .219 .12

26 апреля 2023 г., 05:45:13 
ТЕОРЕТИЧЕСКАЯ

И МАТЕМАТИЧЕСКАЯ

ФИЗИКА

Том 125, № 2

ноябрь, 2000

(C) 2000 г. $\quad$ В. А. Вшивцев ${ }^{*}$, А.В. Прокопов ${ }^{\dagger}$, А.В. Татаринцев ${ }^{\ddagger}$

\section{НЕКОТОРЫЕ ОСОБЕННОСТИ ТЕРМОДИНАМИКИ НЕЛИНЕЙНЫХ КЛАССИЧЕСКИХ СИСТЕМ}

Получены термодинамические характеристики идеального газа двухатомных молекул, межатомное взаимодействие в которых нелинейно. Задача решена для широкого класса потенциалов взаимодействия. Рассмотрение ведется на классическом уровне, что допустимо в области достаточно высоких температур. Показано, что нелинейность взаимодействия может приводить к существенным особенностям свойств газа.

\section{1. ВВЕДЕНИЕ}

Термодинамические характеристики различных физических систем зависят от межмолекулярных и внутримолекулярных взаимодействий в них. Отклонение потенциала взаимодействия от гармонического или необходимость учета дополнительных межмолекулярных сил приводит порой к сушественным изменениям свойств термодинамических величин, таких, например, как теплоемкость, химический потенциал, термодинамическое среднее размера молекул (длины связи) и т.п. Кроме этого, по указанным характеристикам легко судить о числе возбужденных степеней свободы в различных областях температуры, о возможности квазиклассического описания взаимодействия частиш системы и о некоторых других свойствах $[1,2]$.

В данной работе рассмотрены свойства классических термодинамических систем с потенциалом взаимодействия сушественно ангармонического типа. Учет нелинейности сил взаимодействия проводится точно, а не по теории возмушений, что позволяет получить ряд интересных свойств нелинейных систем в области промежуточных температур.

\footnotetext{
* Московский государственный университет, Москва, Россия

${ }^{\dagger}$ Московский государственный институт радиотехники, электроники и автоматики (технический университет), Москва, Россия

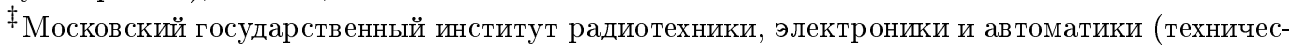
кий университет), Москва, Россия. E-mail: avt@imamod.ru
} 
Наиболее известным примером ангармонического взаимодействия является потенциал

$$
U(x)=U_{0}\left[\left(\frac{x}{d}\right)^{2}-1\right]^{2},
$$

используемый, например, в теории водородной связи воды, где с его помошью удается учесть не только нелинейный характер взаимодействия, но и (при решении задачи на квантовом уровне) процессы переноса протона [3, 4]. Квантово-механическое рассмотрение подобной задачи обнаруживает тонкое расщепление энергетических уровней туннелирующего протона в силу вырожденности энергетического спектра для такого потенциала, что создает определенные трудности при построении термодинамической теории этого эффекта. В то же время вклад подобных эффектов существен лишь в узкой области низких температур и здесь учтен не будет.

Ниже будут рассмотрены термодинамические свойства "идеального газа", состояшего из попарно связанных атомов с потенциалом межатомного взаимодействия типа (1) (аналог водородной связи молекул воды). Будем пренебрегать взаимодействием пар друг с другом, а также эффектами диссоциации и ионизации молекул, полагая, что рассматриваемый интервал температур лежит ниже характерных температур диссоциации. Положим также, что взаимодействие элементов пары может быть рассмотрено на классическом уровне с использованием стандартного распределения Больцмана. Исследуем только вклад, соответствуюший взаимодействию элементов пары, считая, что врашательные степени свободы и поступательное движение пары в целом могут быть учтены стандартным образом [1].

\section{2. ОДНОМЕРНАЯ ЗАДАЧА}

В качестве первого шага рассмотрим одномерную задачу в безразмерных переменных с гамильтонианом $H_{r}(p, x)=p^{2}+U_{r}(x)$, где потенциал взаимодействия

$$
U_{r}(x)=\left(x^{r}-a^{r}\right)^{2}
$$

имеет при четных $r$ два минимума в точках $\pm a$, а при нечетных $r$ - один минимум в точке $a>0$, при этом $U_{\min }=0$. Отметим, что при $r=1$ рассматриваемый потенциал описывает стандартное гармоническое взаимодействие со смешенным средним значением переменной $\langle x\rangle=a$, а при $r=2$ является потенциалом типа (1).

Вклад взаимодействия пары в статистическую сумму определяется статистическим интегралом

$$
Z=\operatorname{Sp} \exp [-\beta H(p, x)]=\iint \frac{d x d p}{2 \pi} e^{-\beta H(p, x)},
$$

где $\beta=1 / T$ - обратная температура, а $H(p, x)$ - гамильтониан. В частности, для потенциала (2), вычисляя соответствуюший интеграл (3), получим

$$
Z_{r}=C_{r}^{(0)} b^{-\frac{r+1}{2 r}} e^{-b} \Phi(b),
$$


где

$$
\Phi(b)=C_{r}^{(1)} M\left(\frac{1}{2 r} ; \frac{1}{2} ; b\right)+2 \sqrt{b} C_{r}^{(2)} M\left(\frac{r+1}{2 r} ; \frac{1}{2} ; b\right)
$$

а $M$ - вырожденная гипергеометрическая функция (функция Куммера) в обозначениях, принятых в книге [5] (см. также [6]). Значения постоянных при $r>1$ имеют следующий вид:

$$
\begin{aligned}
C_{r}^{(0)} & =\frac{a^{r+1}}{\pi 2^{1+\frac{1}{r}}} \Gamma\left(\frac{r+1}{r}\right), \\
C_{r}^{(1)} & =2 \cos \frac{\pi}{2 r} \Gamma\left(\frac{r-1}{2 r}\right), \\
C_{r}^{(2)} & =\left[1+(-1)^{r}\right] \sin \frac{\pi}{2 r} \Gamma\left(\frac{2 r-1}{2 r}\right),
\end{aligned}
$$

а переменная $b=\beta a^{2 r}$ связана с температурой. Отметим, что для нечетных $r$ коэффициент $C_{r}^{(2)}=0$, а при $r=1$ выражение для $C_{r}^{(1)}$ не определено, но может быть заменено соответствующим предельным значением.

Статсумма при $r=2$ может быть получена с использованием частного значения функции Куммера и будет иметь более простой вид

$$
Z_{2}=\frac{a^{3}}{4} \sqrt{\frac{\pi}{b}} e^{-\frac{b}{2}}\left[I_{-\frac{1}{4}}\left(\frac{b}{2}\right)+I_{\frac{1}{4}}\left(\frac{b}{2}\right)\right] .
$$

Используя выражение (4) для статсуммы, легко получить соответствующие вклады в плотность свободной энергии $f \equiv F / N=-T \ln Z$ и теплоемкость

$$
c_{v}=-T \frac{\partial^{2} f}{\partial T^{2}}
$$

для произвольных значений $r$,

$$
c_{v}(r)=\frac{r+1}{2 r}+\frac{\Phi_{1}(b)}{4 r \Phi(b)}-\left[\frac{\Phi_{2}(b)}{2 r \Phi(b)}\right]^{2} .
$$

Функции $\Phi_{1,2}(b)$ в формуле (6) определены следующим образом:

$$
\begin{aligned}
\Phi_{1}(b)= & C_{r}^{(1)}\left[M\left(\frac{1}{2 r} ; \frac{1}{2} ; b\right)+(2 b-1) M\left(\frac{2 r+1}{2 r} ; \frac{1}{2} ; b\right)\right]+ \\
& +2 \sqrt{b} C_{r}^{(2)}\left[2 b M\left(\frac{r+1}{2 r} ; \frac{3}{2} ; b\right)+r(2 b-1) M\left(\frac{r+1}{2 r} ; \frac{1}{2} ; b\right)\right], \\
\Phi_{2}(b)= & C_{r}^{(1)}\left[M\left(\frac{2 r+1}{2 r} ; \frac{1}{2} ; b\right)-M\left(\frac{1}{2 r} ; \frac{1}{2} ; b\right)\right]+2 \sqrt{b} C_{r}^{(2)} r M\left(\frac{r+1}{2 r} ; \frac{1}{2} ; b\right) .
\end{aligned}
$$




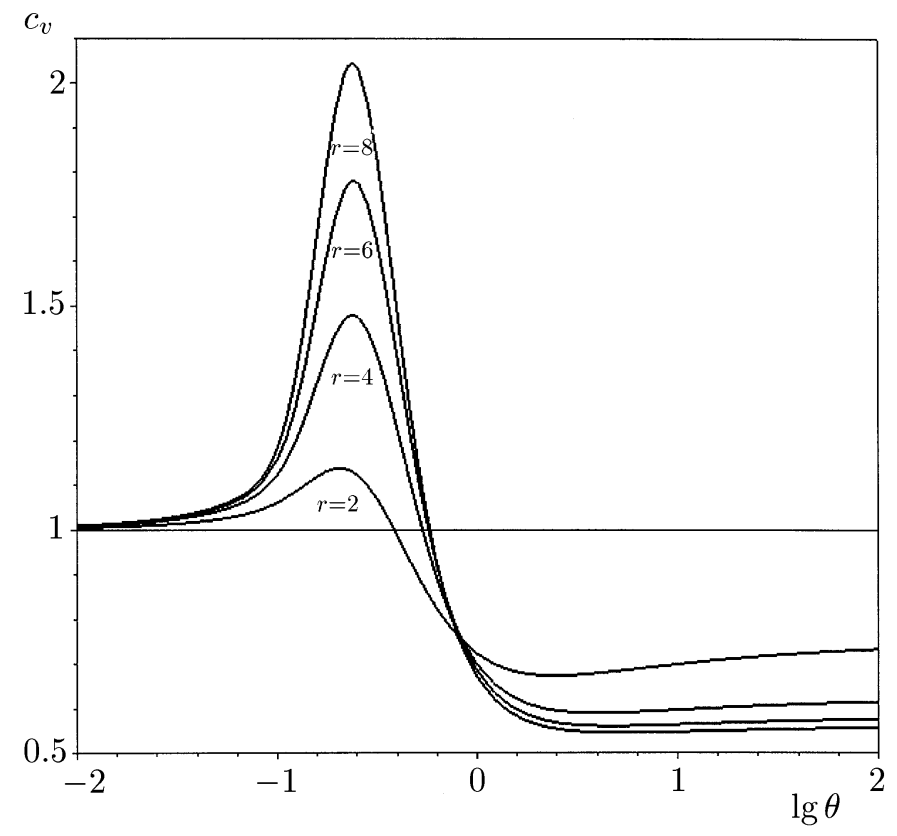

Рис. 1

Зависимость теплоемкости от величины $\theta \equiv 1 / b=T / a^{2 r}$, связанной с температурой $T$, приведена на рис. 1 (четные $r$ ) и на рис. 2 (нечетные $r$ ). Для нечетных значений $r$ коэффициент $C_{r}^{(2)}=0$, и выражение для теплоемкости (6) сушественно упрошается:

$$
c_{v}(r)=\frac{1}{4 r^{2}}\left[\left(2 r^{2}+3 r-1\right)+(2 r b-r+2) \Upsilon(b)-\Upsilon^{2}(b)\right],
$$

где $r=1,3,5, \ldots$, а функция $\Upsilon(b)$ имеет вид

$$
\Upsilon(b)=\frac{M\left(\frac{2 r+1}{2 r} ; \frac{1}{2} ; b\right)}{M\left(\frac{1}{2 r} ; \frac{1}{2} ; b\right)} .
$$

Предельные значения теплоемкостей, следующие из формул (6), (7), одинаковы для всех $r$ в области низких температур:

$$
c_{v}=1+O(\theta), \quad \theta \ll 1,
$$

а для больших значений параметра $\theta \gg 1$ главная асимптотика статсуммы и теплоемкости определяется старшим членом полиномиального потенциала взаимодействий $U_{r}(x) \sim x^{2 r}$ гамильтониана $H_{r}=p^{2}+U_{r}(x)$, отвечающим за сходимость интеграла $(3)$,

$$
c_{v}=\frac{r+1}{2 r}+O\left(\theta^{-2}\right), \quad \theta \gg 1 \text {. }
$$

Для “затравочного" потенциала взаимодействия $V(x)=x^{2 r}$ значение теплоемкости будет равно $(r+1) / 2 r$. В то же время в области низких температур $\theta \ll 1$ (но достаточно 


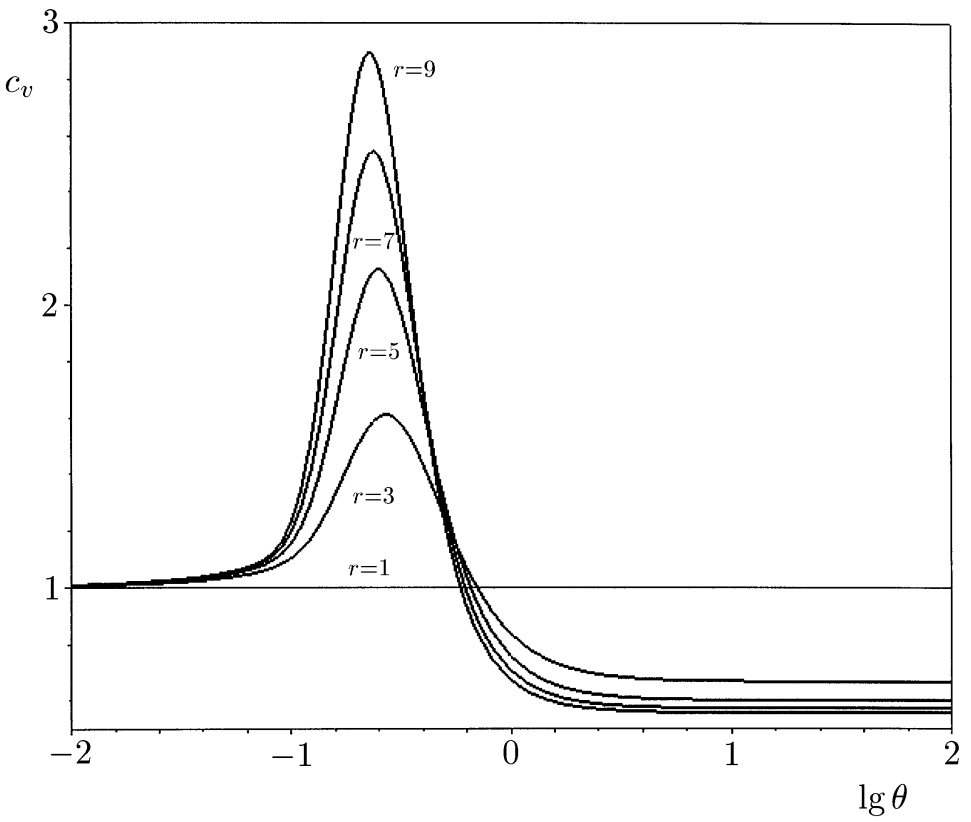

Рис. 2

высоких, чтобы не учитывать квантовые эффекты и различия в статистике) асимптотика $c_{v}$, как это следует из (8), не зависит от $r$. Отметим, что значение теплоемкости в области промежуточных температур имеет локальный максимум, в окрестности которого $c_{v}$ превосходит стандартное значение $c_{v}^{\text {гарм }}=1$ на величину тем большую, чем больше параметр нелинейности потенциала $r$. Для четных $r$ температурная зависимость теплоемкости имеет также локальный минимум.

Подобное поведение теплоемкости идеального газа с ангармонической колебательной степенью свободы молекулы представляет определенный интерес в связи с существованием вешеств с аномальным поведением этой термодинамической характеристики. Возможно, их свойства связаны именно с сушественно нелинейной природой взаимодействия.

Рассмотрим также некоторые интересные особенности поведения термодинамических средних для газа с потенциалом взаимодействия типа (2). Положим, что усреднение производится стандартным статистическим образом:

$$
\left\langle x^{k}\right\rangle_{\beta}=\frac{1}{Z} \operatorname{Sp}\left[x^{k} e^{-\beta H}\right] .
$$

Введем безразмерную переменную, связанную со средним значением, определенным в (10),

$$
\mathfrak{M}_{k}(r, \beta)=\frac{\left\langle x^{k}\right\rangle_{\beta}}{a^{k}}
$$

Будем называть ее моментом соответствующего порядка $k$. Величина $\mathfrak{M}_{1}$ описывает среднее значение координаты (в единицах масштаба $a$ ) и для симметричных потенциалов типа (2) с четным параметром нелинейности $r=2,4,6, \ldots$ обрашается в нуль. Для 


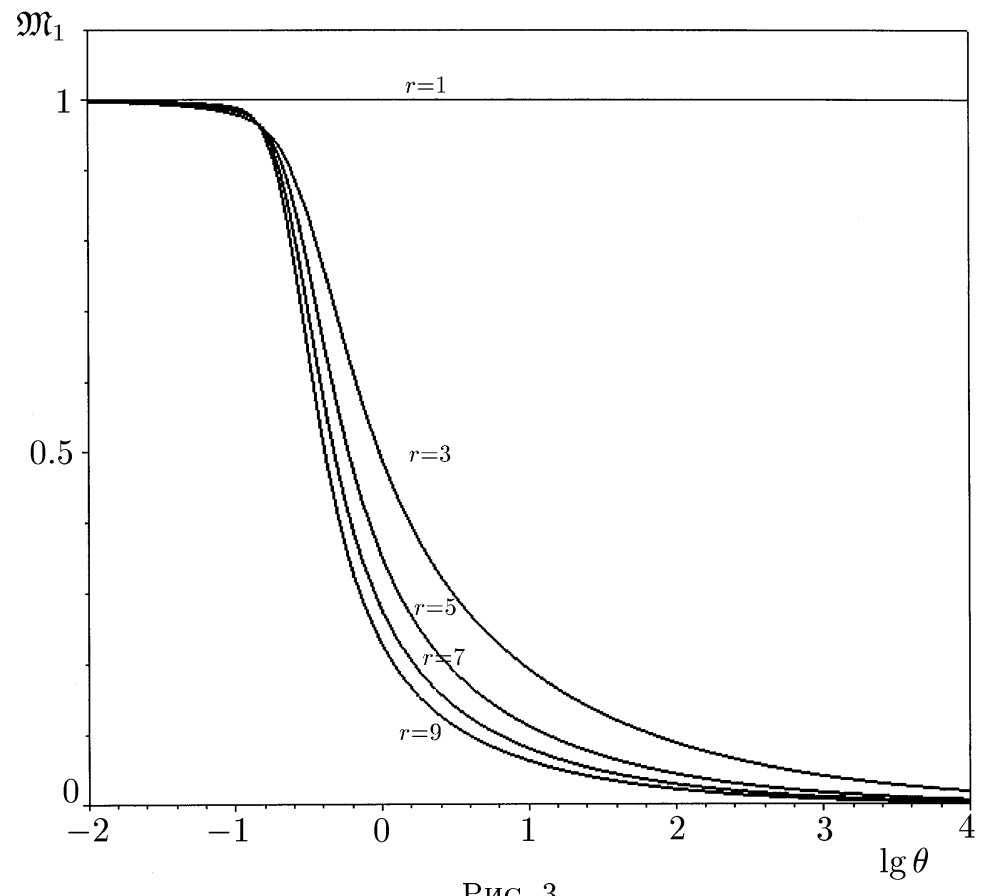

нечетных $r$ потенциал несимметричен, и поэтому $\mathfrak{M}_{1} \neq 0$. Для $r=1$ значение $\mathfrak{M}_{1}=1$, а для $r=3,5,7, \ldots$ из $(10)$ получим

$$
\mathfrak{M}_{1}(r, b)=N_{0}(r) b^{\frac{r-1}{2 r}} \frac{M\left(\frac{r+2}{2 r} ; \frac{3}{2} ; b\right)}{M\left(\frac{1}{2 r} ; \frac{1}{2} ; b\right)} .
$$

Здесь коэффициент $N_{0}(r)$ не зависит от температуры и определяется следующим образом:

$$
N_{0}(r)=2^{2-\frac{1}{r}} \sin \left(\frac{\pi}{2 r}\right) \frac{\Gamma\left(\frac{2}{r}\right) \Gamma\left(\frac{r-1}{r}\right)}{\Gamma\left(\frac{1}{r}\right) \Gamma\left(\frac{r-1}{2 r}\right)} .
$$

Значения момента $\mathfrak{M}_{1}$ для различных $r$ в зависимости от температуры (параметр $\theta$ ) приведены на рис. 3 . Из рисунка видно, что с ростом температуры значения $\mathfrak{M}_{1}$ стремятся к нулю. Это связано с тем, что при $T \rightarrow \infty$ асимптотика значения определяется старшей степенью потенциала $U \sim x^{2 r}$, которая является четной. Таким образом, с ростом температуры газа происходит восстановление исходно нарушенной симметрии.

Момент второго порядка $\mathfrak{M}_{2}$ определяет среднее квадратичное значение длины связи при данной температуре. Для гармонического потенциала $r=1$ момент имеет значение $\mathfrak{M}_{2}(b)=1+1 /(2 b)$ и является монотонной функцией температуры. Для остальных значений $r$ (кроме $r=3$ ), производя усреднение (10), получим

$$
\mathfrak{M}_{2}(r, b)=N_{1}(r) b^{-\frac{1}{r}}\left[C_{r}^{(3)} M\left(\frac{3}{2 r} ; \frac{1}{2} ; b\right)+2 C_{r}^{(4)} \sqrt{b} M\left(\frac{r+3}{2 r} ; \frac{3}{2} ; b\right)\right] \Phi^{-1}(b)
$$




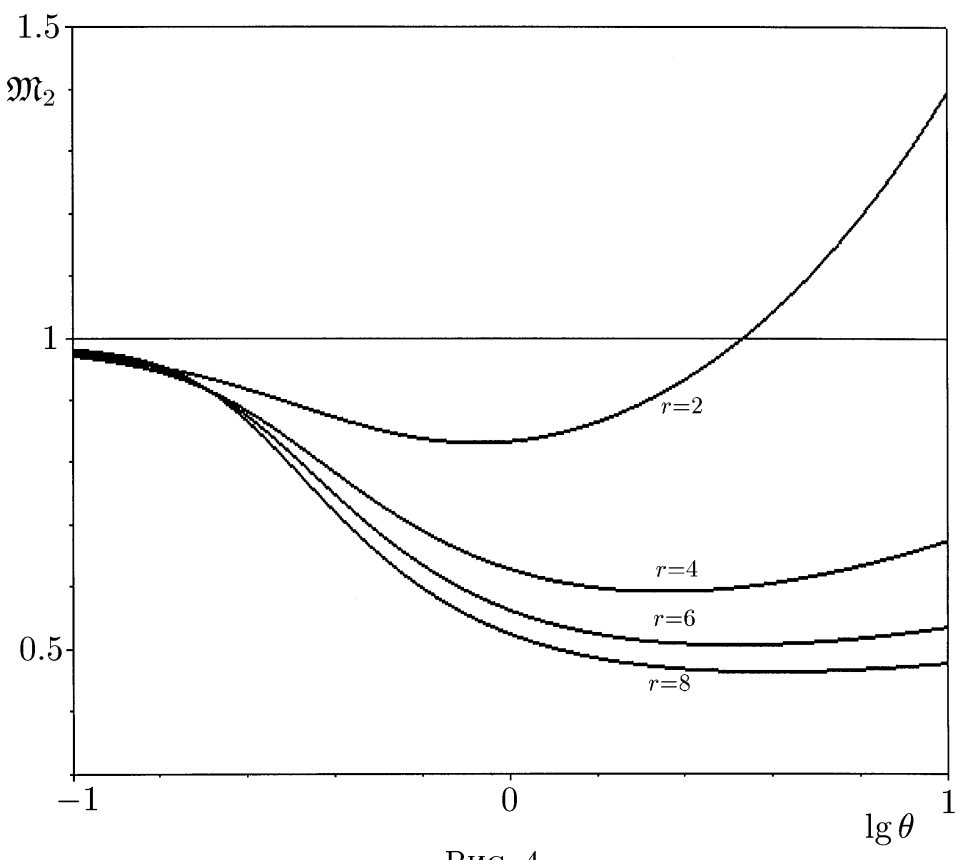

Рис. 4

Здесь функция $\Phi(b)$ определена выше (см. формулу (4)), а постоянные принимают значения

$$
\begin{aligned}
N_{1}(r) & =2^{-\frac{2}{r}} \frac{\Gamma\left(\frac{3}{r}\right)}{\Gamma\left(\frac{1}{r}\right)}, \\
C_{r}^{(3)} & =2 \cos \frac{3 \pi}{2 r} \Gamma\left(\frac{r-3}{2 r}\right), \\
C_{r}^{(4)} & =\left[1+(-1)^{r}\right] \sin \frac{3 \pi}{2 r} \Gamma\left(\frac{2 r-3}{2 r}\right) .
\end{aligned}
$$

Величина $\mathfrak{M}_{2}(b)$ для $r=3$ не может быть определена по формуле (12) из-за неопределенности коэффициента $C_{r}^{(3)}$. Вычисляя данное частное значение, получим

$$
\mathfrak{M}_{2}(3, b)=\frac{2^{\frac{1}{3}} \pi}{\sqrt{3} \Gamma^{2}\left(\frac{1}{3}\right)} b^{-\frac{1}{3}} \frac{e^{b}}{M\left(\frac{1}{6} ; \frac{1}{2} ; b\right)}
$$

Соответствуюшие кривые приведены на рис. 4 (четные $r$ ) и на рис. 5 (нечетные $r$ ). Из их анализа видно, что с уменьшением температуры, как это и следовало ожидать, среднее значение $\left\langle x^{2}\right\rangle^{1 / 2}$ стремится к точкам минимумов потенциалов $U_{r}$. Отметим также, что в отличие от гармонического потенциала $(r=1)$ для всех прочих целых значений параметра нелинейности $(r>1)$ зависимость $\mathfrak{M}_{2}(r, b)$ немонотонна. Величина $\mathfrak{M}_{2}(r, b)$ в случае потенциала $U_{r}$ имеет минимум в области промежуточных температур

6 Теоретическая и математическая физика, т. 125, № 2, 2000 г. 


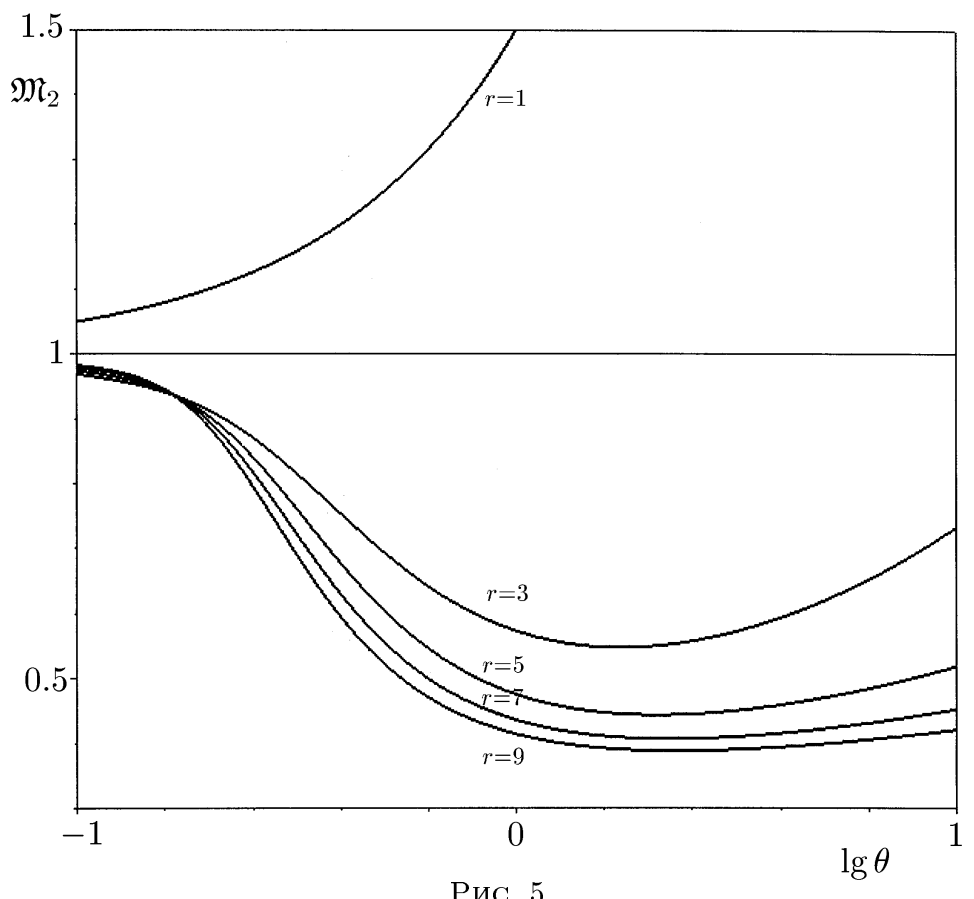

при $\theta=\theta_{\min }(r)$, что является интересной особенностью поведения, присушей только потенциалам, имеющим интервалы различной выпуклости (знаки $U^{\prime \prime}(x)$ различны). Величина эффективной температуры $\theta_{\min }(r)$ возрастает с ростом $r$. В области $\theta<\theta_{\min }$ длина связи двух взаимодействуюших фрагментов пары убывает, что может приводить к аномальному поведению такой характеристики рассматриваемой системы как плотность. При $\theta>\theta_{\min }$ поведение $\mathfrak{M}_{2}(\theta)$ имеет нормальный характер.

\section{3. ДВУМЕРНАЯ ЗАДАЧА}

Рассмотрим теперь систему с двумерным потенциалом, имеющим два симметричных локальных минимума. Подобная задача является обобшением рассмотренной ранее. Некоторые термодинамические аспекты близкой задачи изучались на квантовом уровне в работе [7]. Пусть гамильтониан системы $H=p_{x}^{2}+p_{y}^{2}+U(x, y)$ содержит потенциал взаимодействия

$$
U=\left(x^{2}+y^{2}\right)^{2}-4 a^{2} x y+a^{4}=\rho^{4}-2 a^{2} \rho^{2} \sin 2 \varphi+a^{4},
$$

имеюший два минимума в точках $\rho=a$ и $\varphi=\pi / 4,5 \pi / 4$ и значение $U_{\min }=0$. Вычисляя статистическую сумму по формуле, аналогичной (3), получим

$$
Z=\frac{\sqrt{\pi}}{8} a^{6} b^{-\frac{3}{2}} e^{-\frac{b}{2}} I_{0}\left(\frac{b}{2}\right)
$$


Из (15) следует представление для вклада данных степеней свободы в теплоемкость

$$
c_{v}=\frac{3}{2}+\frac{b^{2}}{4}-\frac{b}{2} \frac{I_{1}\left(\frac{b}{2}\right)}{I_{0}\left(\frac{b}{2}\right)}-\frac{b^{2}}{4}\left[\frac{I_{1}\left(\frac{b}{2}\right)}{I_{0}\left(\frac{b}{2}\right)}\right]^{2} .
$$

Вид соответствуюшей зависимости $c_{v}(\theta)$, определяемый выражением $(16)$, где $\theta \equiv 1 / b=$ $T / a^{4}$, в целом аналогичен температурной зависимости (6) для одномерного потенциала (см. рис.1 и 2). Имеется также точка максимума теплоемкости, а предельные значения отличаются от соответствуюших значений (8), (9) в два раза (по числу степеней свободы):

$$
\begin{aligned}
& c_{v}=2+O(\theta), \quad \theta \ll 1, \\
& c_{v}=\frac{3}{2}+O\left(\theta^{-2}\right), \quad \theta \gg 1 .
\end{aligned}
$$

Средние значения квадратов координат $\left\langle x^{2}\right\rangle_{\beta},\left\langle y^{2}\right\rangle_{\beta}$ и их произведения $\langle x y\rangle_{\beta}$ вычисляются аналогичным (10) образом. Из симметрии потенциала следует, что $\left\langle x^{2}\right\rangle_{\beta}=$ $\left\langle y^{2}\right\rangle_{\beta}$, и поэтому приведем лишь два выражения:

$$
\begin{aligned}
& \mathfrak{M}_{x x}(b) \equiv \frac{\left\langle x^{2}\right\rangle_{\beta}}{a^{2}}=\frac{e^{\frac{b}{2}}}{\sqrt{4 \pi b} I_{0}\left(\frac{b}{2}\right)}, \\
& \mathfrak{M}_{x y}(b) \equiv \frac{\langle x y\rangle_{\beta}}{a^{2}}=\frac{1}{4}\left[1+\frac{I_{1}\left(\frac{b}{2}\right)}{I_{0}\left(\frac{b}{2}\right)}\right] .
\end{aligned}
$$

Анализируя функциональную зависимость моментов и теплоемкости, легко получить, что и в двумерном случае поведение термодинамических величин сохраняет все особенности, присушие одномерному случаю. Имеется область температур, в которой значение теплоемкости превосходит соответствующее "гармоническое" значение $c_{v}^{\text {гарм }}=2$, а также сушествует точка минимума $\mathfrak{M}_{x x}(\theta)$. Отметим, что с ростом температуры величина $\mathfrak{M}_{x y}(\theta)$ уменьшается до своего предельного значения $1 / 4$ (доля частиц с большой коррелящией координат $x, y$ падает). При уменьшении температуры средние значения определяются точками минимумов потенциала (14) $x_{\min }=y_{\min }= \pm a / \sqrt{2}$, что связано в классическом подходе с "опусканием" частишы на дно потенциальной ямы при уменьшении случайных температурных колебаний.

\section{4. ТРЕХМЕРНАЯ ЗАДАЧА}

Приведем также некоторые результаты, иллюстрируюшие возможность вычисления соответствуюших термодинамических величин для наиболее реалистического случая трехмерного потенциала, имеюшего два локальных минимума, разделенных потенциальным барьером. Потенциал взаимодействия выберем в виде

$$
U=\left(R^{2}+a^{2}\right)^{2}-4 a^{2} z^{2}=R^{4}-2 a^{2} R^{2} \cos 2 \vartheta+a^{4} .
$$


Интегрирование по углу $\vartheta$ в сферических координатах проще всего провести, используя разложение

$$
e^{t \cos 2 \vartheta}=I_{0}(t)+2 \sum_{k=1}^{\infty} I_{k}(t) \cos 2 k \vartheta .
$$

Вычисляя интеграл по $R$, получим следующее выражение для статсуммы:

$$
Z=\frac{a^{9} b^{-\frac{9}{4}}}{8 \sqrt{\pi}} e^{-b}\left[\Gamma\left(\frac{3}{4}\right) M\left(\frac{3}{4} ; 1 ; b\right)-2 \sum_{k=1}^{\infty} \frac{\Gamma\left(\frac{2 k+3}{4}\right) b^{\frac{k}{2}}}{k !\left(4 k^{2}-1\right)} M\left(\frac{2 k+3}{4} ; k+1 ; b\right)\right] .
$$

Используя асимптотику функций Куммера в области высоких температур при $b \equiv a^{4} / T \rightarrow 0$, получим $Z \sim b^{-9 / 4}$, и, следовательно, предельное значение теплоемкости будет равно $9 / 4$, что составляет по $3 / 4$ на каждое измерение с ангармоническим взаимодействием и соответствует величине, полученной ранее.

\section{5. КВАНТОВАЯ ЗАДАЧА}

Подробное рассмотрение квантовой задачи (а также учет статистических особенностей) планируется авторами провести в отдельной работе. В этом разделе укажем лиш на некоторые аналогии, имеющиеся между классической и квантовой задачами для потенциалов типа (2).

Статистическая сумма в квантовом случае будет иметь вид

$$
Z(\beta, \delta)=\sum_{n} e^{-\beta \varepsilon_{n}}
$$

Здесь $\varepsilon_{n}$ - собственное значение гамильтониана $H_{r}=p^{2}+U_{r}(x)$. Отметим, не вдаваясь в подробности $[8,9]$, что спектр собственных значений энергии для потенциала с асимптотикой $U_{r}(x) \sim x^{2 r}$ имеет для больших значений $n$ асимптотику

$$
\varepsilon_{n} \sim(2 n+1)^{\delta}, \quad \delta=\frac{2 r}{r+1}
$$

В высокотемпературном пределе имеет значение именно эта асимптотика. Произведем суммирование ряда для статсуммы с помощью стандартного преобразования Меллина:

$$
Z_{\mathrm{M}}(s, \delta) \equiv \int_{0}^{\infty} d \beta \beta^{s-1} Z(\beta, \delta)=\left(1-2^{-\delta s}\right) \Gamma(s) \zeta(\delta s)
$$

Вычисляя, далее, оригинал с помощью теории вычетов, найдем

$$
Z(\beta, \delta)=\frac{1}{2} \Gamma\left(\frac{1}{\delta}\right) \beta^{-\frac{1}{\delta}}+\sum_{k=1}^{\infty} \frac{(-1)^{k}}{k !}\left(1-2^{-\delta k}\right) \zeta(\delta k) \beta^{k} .
$$

В области высоких температур $\beta \equiv 1 / T \rightarrow 0$ асимптотика статистической суммы имеет вид $Z \sim \beta^{-(r+1) / 2 r}$, что приводит к значению теплоемкости

$$
c_{v}=\frac{r+1}{2 r}+O(\beta)
$$

Предельное значение теплоемкости, таким образом, совпадает с величиной (9), полученной в классическом подходе. 


\section{6. ЗАКЛЮЧЕНИЕ}

Выше были исследованы некоторые термодинамические свойства идеального газа молекул, имеющих существенно нелинейную колебательную степень свободы. Поведение полученных таким образом термодинамических характеристик газа имеет важные особенности. Рассмотрение ведется на классическом уровне, что оправданно в области промежуточных температур в силу массивности молекулярных фрагментов. Как было показано, для одномерной и двумерной задач вклад $c_{v}$ нелинейного межатомного взаимодействия в теплоемкость в области промежуточных температур может превосходить аналогичный вклад для случая гармонического взаимодействия на величину тем большую, чем больше параметр нелинейности потенциала $r$. Кроме того, характерной особенностью для случая ангармонического взаимодействия с потенциалами (2), (14), имеюшими два минимума, является немонотонность температурной зависимости среднего квадратичного значения координаты $\left\langle x^{2}\right\rangle_{\beta}$, соответствующего в данной постановке задачи эффективной температурной длине связи молекулярных фрагментов. Наличие минимума величины $\mathfrak{M}_{2}(\theta)$ может приводить к аномальному поведению плотности вешества (особенно в жидкой фазе) с ростом температуры.

Отметим также, что высокотемпературная асимптотика $\Omega$-потенциала пропорциональна $T^{5 / 2}$ для рассмотренного двумерного потенциала (14) и отличается от стандартного закона Стефана-Больцмана $\left(\sim T^{3}\right)$ [8]. Такое отклонение может быть вызвано ограничением области применимости рассмотренной модели. В области высоких температур процессы диссоциации будут вести к разрыву всех межмолекулярных и внутримолекулярных связей, что означает полное исчезновение данных степеней свободы. Таким образом, все полученные результаты справедливы, строго говоря, лишь в области промежуточных температур, не превосходящих характерной температуры диссоциации.

\section{Список литературы}

[1] Л. Д. Ландау, Е. М. Лифииц. Статистическая физика. Ч. 1. М.: Наука, 1995.

[2] Р. Балеску. Равновесная и неравновесная статистическая механика. Т. 1, 2. М.: Мир, 1978.

[3] В. В. Антонченко. Физика воды. М.: Наука, 1986.

[4] А.С. Виивцев, А.Ф. Королев, К.Г. Круглов, А. В. Татаринцев. Изв. вузов. Физика. 1997. № 10. C. 37-41.

[5] Справочник по специальным функциям. Ред. М. Абрамовиц, И. Стиган. М.: Наука, 1979.

[6] Г. Бейтмен, А. Эрдейи. Высшие трансцендентные функции. Т. 2. М.: Наука, 1966.

[7] M. E. ШІтарев. Механизмы туннелирования протона в теории водородной связи. Дипломная работа. М.: Физ. фак. МГУ, 1999.

[8] В.П. Барашев, В. В. Белов, А.С. Виивцев, А.Г. Кисунько. ТМФ. 1998. Т. 116. № 3. C. $431-441$.

[9] А. С. Вичвцев, Н. В. Норин, В. Н. Сорожин. ТМФ. 1996. Т. 109. № 1. С. 107-123.

Поступила в редакцию 22.III.2000 г., после доработки 18.V.2000 г. 